ITC $1 / 48$

Journal of Information Technology and Control

Vol. 48/ No.1/2019

pp. $5-17$

DOI 10.5755/j01.itc.48.1.22246
A Conceptual Framework for Modelling Spatial Relations

Received 2018/12/10
Accepted after revision 2019/01/28

\title{
A Conceptual Framework for Modelling Spatial Relations
}

\section{Eliseo Clementini}

Department of Industrial and Information Engineering and Economics; University of L'Aquila; via G. Gronchi 20, I-67100 L’Aquila, Italy; phone: +39 0862 434438; fax: +39 0862 434403;

e-mail: eliseo.clementini@univaq.it

Corresponding author: eliseo.clementini@univaq.it

Various approaches lie behind the modelling of spatial relations, which is a heterogeneous and interdisciplinary field. In this paper, we introduce a conceptual framework to describe the characteristics of various models and how they relate each other. A first categorization is made among three representation levels: geometric, computational, and user. At the geometric level, spatial objects can be seen as point-sets and relations can be formally defined at the mathematical level. At the computational level, objects are represented as data types and relations are computed via spatial operators. At the user level, objects and relations belong to a context-dependent user ontology. Another way of providing a categorization is following the underlying geometric space that describes the relations: we distinguish among topologic, projective, and metric relations. Then, we consider the cardinality of spatial relations, which is defined as the number of objects that participate in the relation. Another issue is the granularity at which the relation is described, ranging from general descriptions to very detailed ones. We also consider the dimension of the various geometric objects and the embedding space as a fundamental way of categorizing relations.

KEYWORDS: Spatial relations, spatial data, geographic data, geometric invariants, topological relations, projective relations, metric relations.

\section{Introduction}

Spatial relations have been an active research subject for thirty years. The theme is interdisciplinary and has attracted the interest of the different scientific communities, not only in computer science, but in linguistics [55], philosophy [13], and psychology [53]. In computer science, different domains, such as spatial databases [49], Geographic Information Systems (GIS) [38], image databases [4, 10], and Qualitative
Spatial Reasoning (QSR) [31, 46] involve research on spatial relations. Over the years, various models of spatial relations have been defined: some have been transferred to current technology, while others remain theoretical contributions. This article is an attempt to define a conceptual framework for comparing these models in a unifying context. The framework is important to understand the importance of 
each model and the relationships between different approaches and solutions. In addition to including the state of the art, the framework can help to identify the aspects that have been less investigated so far and can still be the subject of future research. The conceptual framework is structured according to "orthogonal axes", that is, according to independent characteristics that allow us to define a categorization of spatial relations (Figure 1). Overall, we propose a categoriza- tion of spatial relations according to six axes: the first is the level of representation. The second relates to the properties of the geometric space. The third is the cardinality of relations. Then, we consider granularity. The next axis concerns the type of objects and their dimension and the latter considers the dimension of the embedding space. In the following, each axis is discussed in a separate section.

Figure 1

The conceptual framework of spatial relations structured in six orthogonal axes

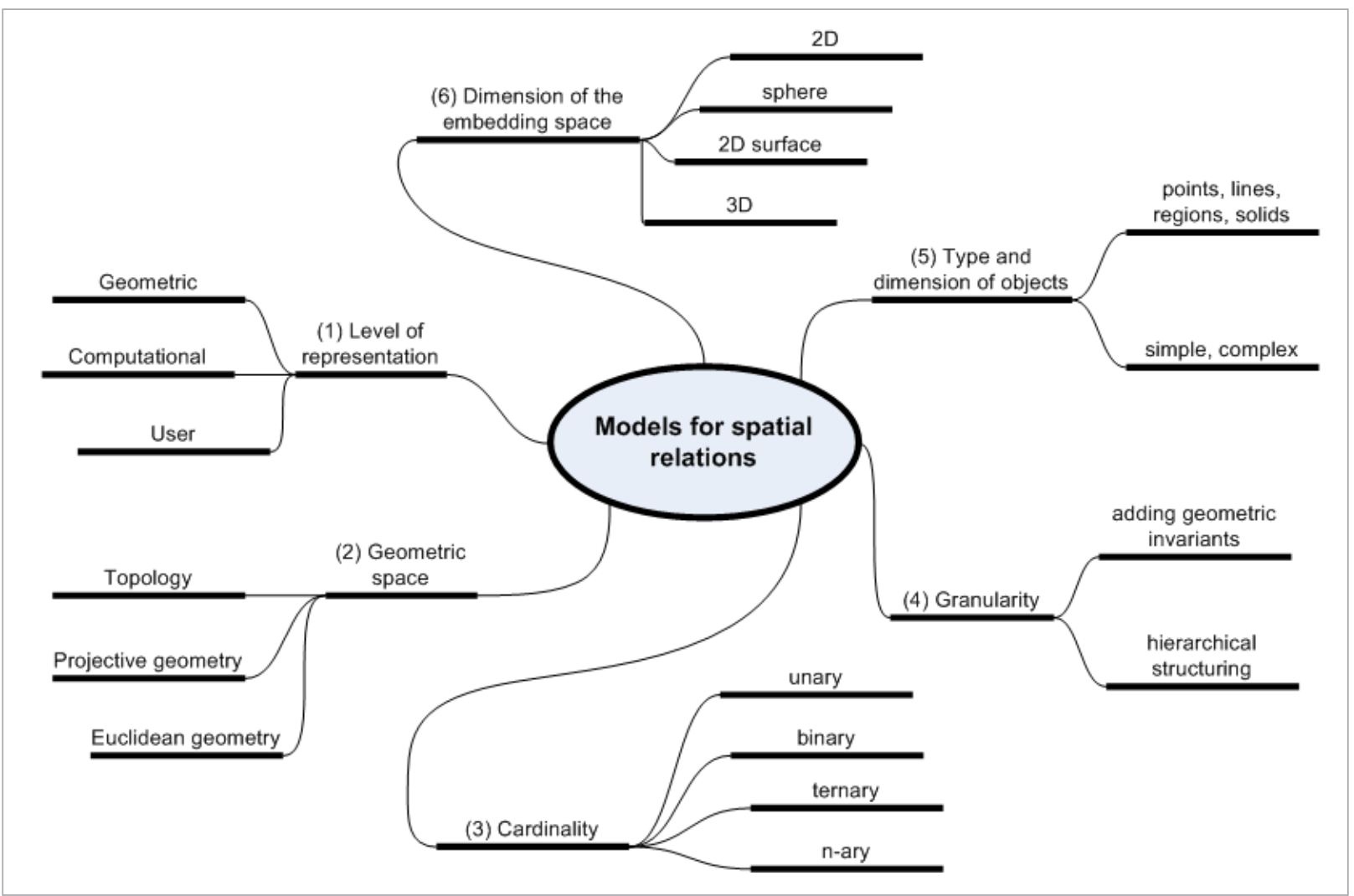

\section{Levels of Representation}

Spatial relations can be categorized according to three different levels of representation: the geometric level, the computational level, and the user level. The geometric level is an abstract representation in mathematical terms of objects, where spatial relations between them are defined by specific geometric properties: for example, in the model of the 4 intersections (4IM) [35], topological relations are defined on the empty and non-empty values of the boundary and interior intersections of the two objects. The geometric level can be considered the most primitive level for the study of spatial relations, since it allows to find formal definitions. The other two levels will relate to the definition of a spatial relation at the geometric level.

At the computational level, spatial objects are represented as spatial data types and spatial relations be- 
tween objects must be computed by spatial operators. Although the geometric level can be considered, by definition, without errors, the representation of spatial objects at the computational level is intrinsically affected by approximation. Real objects are represented with a simplified model: for example, the historical classification of representations in GIS is in raster and vector representations [32]. In raster models, the approximation is given by the size of the cells, whereas in vector models the approximation is given by the number of points representative of a curve. In the field of spatial database systems, the vector approach is the most widespread: various proposals of database systems supporting spatial data and spatial relations have been developed (e.g., [12, 44, 48, 52]). The issues that are considered at the computational level are mainly related to the system performance in computing relations. The recent trend is that spatial database systems adhere to the specifications of the Open Geospatial Consortium (OGC): systems such as Oracle, IBM DB2, and PostgreSQL base their definition of data types and spatial operators on OGC specifications for SQL [57]. For example, the following is a definition of an operator to calculate a topological relation:

Crosses (g1 Geometry, g2 Geometry): Integer

The above operator finds a corresponding definition at the geometric level in the "cross" relation of the Calculus-Based Method (CBM) [27]. The set of relations that are part of the OGC specification is limited to topological relations. Further work is needed to define different types of spatial relations that could be included in the standard, such as cardinal directions: several proposals have been published in the literature, e.g., $[18,47]$, to represent cardinal directions between extended objects.

The problem of approximation in spatial data models and the degree of uncertainty that results from this is a question of ongoing research and is far from being solved. In some approaches, to continue to use the same models for topological relations defined at the geometric level, new data types have been defined which are able to model the uncertainty in the boundary of objects as a two-dimensional band around the object itself $[15,25,30]$. In addition, there may be several descriptions of the same object according to different levels of accuracy.
The raster model is much more used in the field of image databases. The computation of spatial relations in image databases is less direct than in the case of spatial databases where each space object is represented separately, and the relations are computed using geometric algorithms. In raster models, to compute relations, first the objects must be extracted, and then spatial relations are evaluated with quantitative methods, e.g., [4]. No particular effort has been made to date in adapting quantitative methods to assess spatial relations with qualitative models of spatial relations [14]. In some cases, the models of spatial relations have been extended to work with raster data [39, 67]. Other approaches to image databases are devoted to establishing blurred measurements of qualitative spatial relationships understood as linguistic expressions [1]. These combinations of fuzzy quantitative models [10] or probabilistic [33] with qualitative models for evaluating spatial relations in images are also called hybrid models.

At the user level, objects and spatial relations are linked to a specific application context. We can assume that these concepts can be defined in a spatial ontology: there are various approaches in the literature, for example, in the context of urban information systems [3] or more generally in conceptual modeling [58]. Spatial relations at this level depend strongly of various factors, such as the peculiarity of the domain, vagueness and imprecision of user terms, and the variability of terms in different countries and natural languages. To give an example, consider a navigation context: a GPS-equipped smartphone is able to give indications on the buildings that are visible by the user, who moves with a car in the city. This could be expressed with the following query: "What are the names of the buildings that are to the right and left and forward of the user position, for which there is no other obstacle between them and which are at a reasonable distance? ». Spatial relations used in this example will need to be transferred to the geometric level to find the equivalent mathematical definitions and at the computational level in terms of appropriate geometric types and relations [2].

The definition of spatial ontologies describing the classes of a specific area of application (e.g., navigation systems for tourist information) and the possible spatial relations between the objects of these classes is a question of research. An object at the user level 
(for example, a stream) can have multiple representations at the geometric level, since a river can be represented as a single line or a complex line, or as a two-dimensional region. Therefore, a semantic spatial relations between two streams (for example, one river flows into another watercourse) can be modelled with various geometric relations based on the adopted representations [63]. There are also architectural problems on how to interpret ontology at the user level in terms of data type and operators at the computational level [17].

An important aspect that the user ontology on spatial relations should consider is the context information. For example, a projective relation such as "left_of" has a unique meaning when associated with certain context information. From the point of view of the user, the relations must be combined with the presence of a reference system to remove ambiguities in the meaning of the relation. If the context varies, often the meaning of the relation becomes ambiguous: the expression "the tree to the left of the university", which is unambiguous in a given context, becomes ambiguous if extrapolated from its context (see Figure 2).

\section{Figure 2}

Different interpretations of the term "the tree to the left of the university" according to the context: (a) the left is determined by an intrinsic property of the building, (b) the left is determined by a user's point of view

a

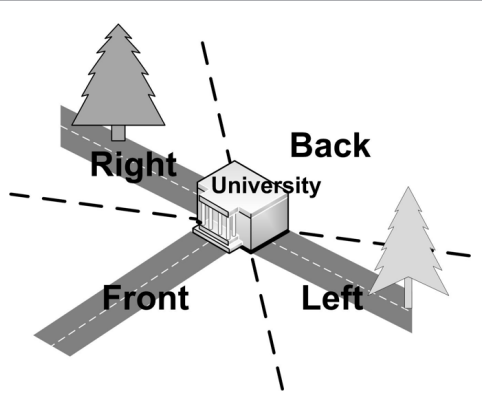

b

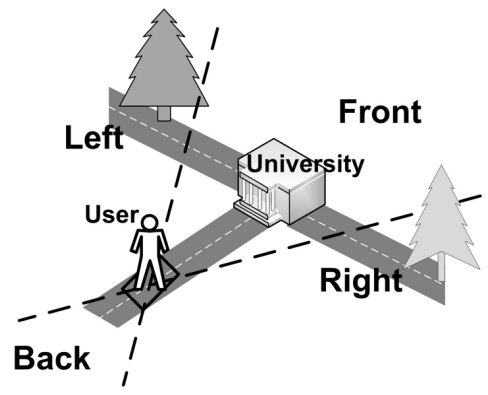

Three types of reference systems are distinguished in the literature [60]: intrinsic reference systems are based on a reference object that determines the origin of the coordinate system as well as its orientation. Extrinsic reference systems can also inherit their origin from a reference object; However, their orientation is determined by external factors such as the direction of movement or by a conventional object used as a reference point. Deictic reference systems involve three objects: a primary object, a reference object, and a point of view. The orientation of the reference system is imposed on the reference object as seen by the point of view. From this basic categorization, the user ontology must describe more specific reference systems as well as the possible spatial relations between object classes. A more refined ontology of frames of reference has been proposed in [18].

\section{Geometric Space Properties}

We propose to classify spatial relations according to three geometric categories: topological, projective, and metric, which are based on the properties of the topological, projective, and Euclidean space, respectively. While topological relations have been widely discussed in the literature, e.g., [22, 27, 29, 37], and the evolution of topological relations has invested all the orthogonal axes proposed in this article, the other two categories are less consolidated. Projective relations are a category of spatial relations that can be described by projective properties of space without resorting to metric properties [20]. Like topological relations, projective relations are qualitative in nature because they do not need specific measures to be explained [38]. In addition, projective relations are more accurate than topological relations and can serve as a basis for describing relations that are not described by topology. Being an intermediate step between metric and topology, projective relations are as varied as "to the right of", "in front of", "between", "along", "surrounded by", "before", "back", "north of", "east of", and so on. Although specific models have been developed for subsets of projective relations, such as cardinal directions [45], the orientation relations [50], and cardinal directions for extended objects [47], we lack a unifying model that is capable of representing all variants of projective relations. A starting point for 
building a general model for projective relations is the 5 -intersection model $[5,19,21]$. This model gives a classification of projective relations between three geometric objects in the plane, distinguishing 34 relations that form a JEPD set (jointly-exhaustive and pair-wise disjoint). The latter property is essential for developing reasoning systems of relations [6,28]. As far as metric relations are concerned, such as the distance between two points, they are normally meant as quantitative relations, but there are qualitative approaches to describe distances that could be further investigated [26].

\section{Figure 3}

The hierarchy of geometries

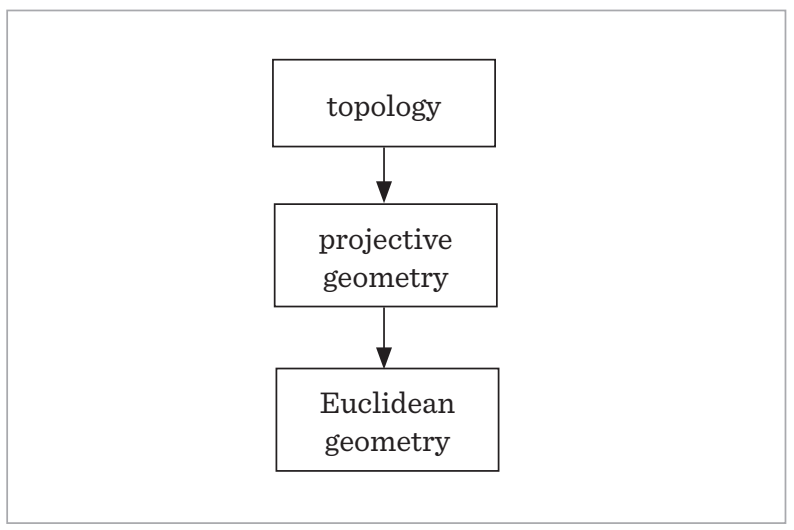

The three spaces are not independent between them, but they are hierarchically linked (see Figure 3). Fol- lowing the axiomatic approach in geometric spaces [54], a space can be built from the previous one by adding more axioms. If a geometric property is evaluated in the topological space, the same property will remain valid in the projective space and the Euclidean space. The opposite is not true: if we can identify a property in the Euclidean space, the same property will not necessarily be valid in the projective space and the topological space. It would be more accurate to say that the property cannot be identified in these spaces. Thereafter, we limit ourselves to an informal description of the properties of the three spaces.

For example, let us consider the topological property "having a hole" in a two-dimensional region, the same object embedded in a projective or Euclidean space will maintain the property to have a hole. This property is topological because in a topological space, we have the formal means to identify it. A topological property is also called a topological invariant, because the property is valid after the application of any topological transformation. More formally, a topological transformation is a bi-continuous transformation, also known as rubber "sheeting" or elastic transformation, i.e., the topological space is a kind of rubber sheet that can be distorted at will as long as you do not make a hole and you do not tear. In addition, a topological relation between two objects such as "overlap" is invariant following a topological transformation.

\section{Table 1}

Categorization of spatial relations by crossing geometric space and cardinality

\begin{tabular}{|c|c|c|c|c|c|}
\hline $\begin{array}{l}\text { Geometric space/ } \\
\text { cardinality }\end{array}$ & \multicolumn{2}{|c|}{ unary } & binary & ternary & $n$-ary \\
\hline topological & $\begin{array}{l}\text { two separate } \\
\text { components }\end{array}$ & one hole & A touch B & overlap & network \\
\hline projective & concave & $\begin{array}{l}\overbrace{\text { four points }}^{0} \\
\text { of order } 0\end{array}$ & A inside concavity of $B$ & (C) B & $\begin{array}{cc}\square & \square \\
\square \quad 0 \\
\square & \square \\
\text { surrounded by }\end{array}$ \\
\hline metric & four angle & $90^{\circ}$ degrees & $\begin{array}{c}\mathrm{A} \\
\text { A bigger than } \mathrm{B}\end{array}$ & $\begin{array}{c}\mathrm{A} \square \\
\square \quad \stackrel{C}{\square} \\
\text { equidistant }\end{array}$ & $\begin{array}{c}\square \square \square \square \\
\square \square \square \square \\
\square \square \square \square \\
\text { grid }\end{array}$ \\
\hline
\end{tabular}


If we consider a projective property as "being a triangle", such a property is true if we include the object in a projective or Euclidean space, but not true in a topological space, since the property cannot be defined in topology: there is no way to distinguish internally such objects (in topology a triangle can be transformed into a circle). Similarly to topological properties, a projective property can be defined inside a projective space by saying that a projective property is a projective invariant, that is, it does not change after a projective transformation. Therefore, a projective transformation is a transformation that preserves the collinearity of three points in space. An example of a projective relation is a point "to the right" of an oriented line. This relation is preserved after a projective transformation.

Finally, if we consider the metric property of a polygon "having a right angle $\left(90^{\circ}\right)$ ", including the object in a projective or topological space, we lose the ability to distinguish this property. A right angle is a characteristic that is preserved after roto-translation, but it will not be preserved after a projective transformation or topological transformation. After a projective transformation, we will still have a polygon, but the measurement of this particular angle will be different from $90^{\circ}$. A summary of the examples for the three geometric types combined with the cardinality axis of the next section is given in Table 1.

\section{Cardinality}

Spatial relations can be classified with respect to their cardinality, i.e., the number of objects involved in the relation. While binary relations are the most commonly admitted, it is useful and, in some cases, necessary to consider other cardinalities. To be complete, we can also consider unary relations, which correspond to geometric properties of single spatial objects. Mathematically, a binary relation between two sets $A$ and $B$ is a subset of the Cartesian product $A \times B$. Topological relations are mainly binary because they are based on the connection property of two objects (two sets of points can either have common parts or not). Rarely, ternary topological relations have been taken into account [40]. Ternary relations involve three objects $A, B$ and $C$. This category is very useful for projective relations, which are based on three-point collinearity (the fundamental geometric invariant in a projective space) [7]. A typical ternary relation is "between" [11]. The results on reasoning mechanisms with ternary relations, such as composition tables [46], have yet to be developed. Qualitative spatial reasoning on ternary relations is a topic of active research $[6,28,66]$. More rarely, in the literature, quaternary relations are envisaged: for example, in order to illustrate the notion of surrounding [59]. In general, when more than three objects are concerned, we talk about $n$-ary relations. In Table 1 , we propose some examples of spatial relations by combining the properties of geometric spaces and cardinality.

\section{Granularity}

Another issue is the degree of granularity with which spatial relations can be described. For example, let us consider a topological relation that expresses the fact that two regions overlap, the same topological relation can be refined with more granularity by specifying the number of distinct parts in the intersection of the regions. For example, in Figure 4, the two cases can be described as an 'overlap' relation. If we consider the number of separate parts, we are able to make a difference between the two cases. Therefore, if we limit ourselves to a more abstract level of granularity, a relation can make a coarse subdivision of possible geometric configurations without distinguishing the more detailed aspects; by adding a more detailed property to the relation, we can subdivide the possible configurations in different more detailed subclasses.

\section{Figure 4}

Effect of adding greater granularity to the "overlap" relation with the number of distinct components of the intersection of regions: (a) equal to 1 ; (b) equal to 2

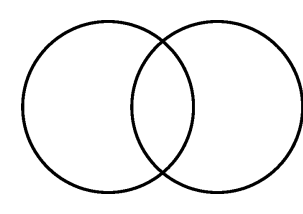

a

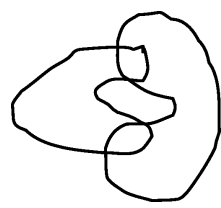

$\mathrm{b}$ 


\section{Figure 5}

The 31 different relations between lines and regions in the DE+9IM. Each box contains the cases belonging to the same case of the DEM

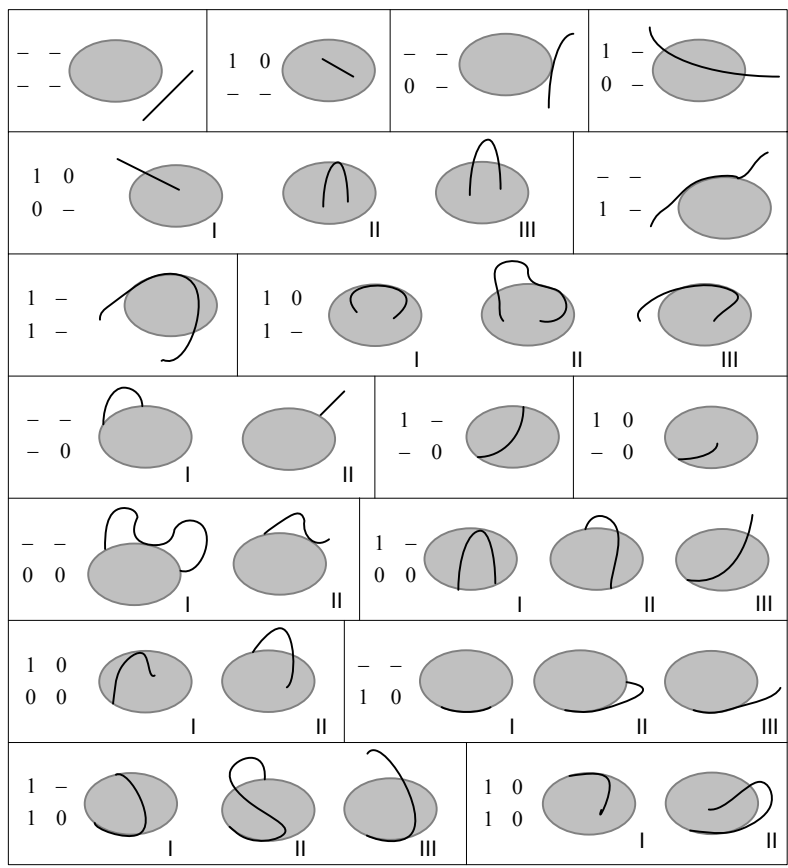

With respect to topological relations, several topological invariants can be considered to add granularity to the relations $[24,36]$. Based on the invariant of the content, the model of 9-intersection (9IM) is an extension of the 4IM with the addition of the concept of external objects, besides the interior and the boundary [37]. An important invariant is the dimension of the parts of the objects, which produced a class of models, called Dimension-Extended, such as the "Dimension-Extended Method » (DEM), the "Calculus-Based Method» (CBM) [27], and the DE+9IM [23]. By expressive power of these models, we will indicate the number of topological relations that the model is able to distinguish. For example, the $\mathrm{DE}+9 \mathrm{IM}$ is expressed as a $3 \times 3$ matrix containing the dimension of the interior $\left(\lambda^{\circ}\right)$, boundary $(\partial \lambda)$, and exterior $\left(\lambda^{-}\right)$of the intersections. The "dim" function returns the highest dimension of the intersection set. The possible values in 2D space are 0, 1 and 2, for non-void intersection of points, lines and regions, respectively, and null (indicated with '_-') for the empty intersection:

$$
\left(\begin{array}{lll}
\operatorname{dim}\left(\lambda_{1} \circ \cap \lambda_{2}{ }^{\circ}\right) & \operatorname{dim}\left(\lambda_{1} \circ \cap \partial \lambda_{2}\right) & \operatorname{dim}\left(\lambda_{1} \circ \cap \lambda_{2}^{-}\right) \\
\operatorname{dim}\left(\partial \lambda_{1} \cap \lambda_{2}{ }^{\circ}\right) & \operatorname{dim}\left(\partial \lambda_{1} \cap \partial \lambda_{2}\right) & \operatorname{dim}\left(\partial \lambda_{1} \cap \lambda_{2}{ }^{-}\right) \\
\operatorname{dim}\left(\lambda_{1}{ }^{-} \cap \lambda_{2}{ }^{\circ}\right) & \operatorname{dim}\left(\lambda_{1}{ }^{-} \cap \partial \lambda_{2}\right) & \operatorname{dim}\left(\lambda_{1}^{-} \cap \lambda_{2}^{-}\right)
\end{array}\right)
$$

This model is capable of distinguishing between 87 real topological relations. In order to illustrate some relations, in Figure 5, we present the sequence of 31 line/region cases for the DE+9IM, grouped by the DEM values (upper left of the DE+9IM matrix).

Regarding projective relations, a similar process can be adopted for the construction of a hierarchy of relations, from the most general to the most specific (Figure 6). For example, the collinearity of three points can be refined into the relations "between" and "nonbetween", and in addition, considering a line orientation, the relation "nonbetween" can be refined in the "before" and "after" relations. The definitions of this set of relations among points are given in Table 2 (excerpt from [19]).

In terms of qualitative metric relations, granularity is based on the number of sub-divisions of the space [26]. For example, in Figure 7(a), the relation between $A$ and $B$ is expressed as "far", since the space is divided into two qualitative regions. In Figure $7(b)$, the same relation is expressed as "medium", since the space is divided into three regions.

\section{Figure 6}

A decision tree for projective relations between points

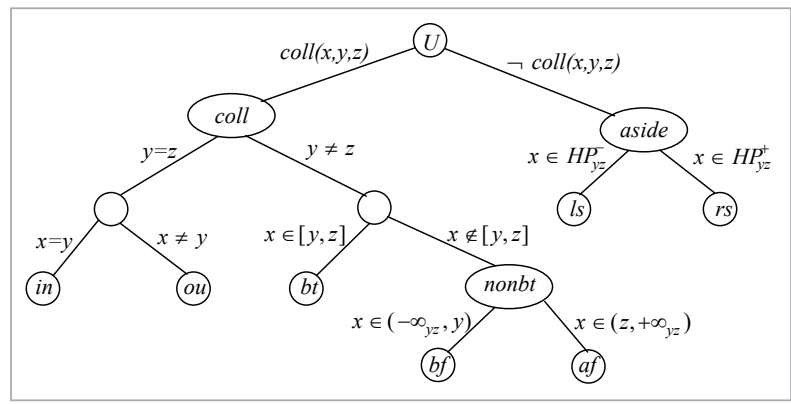

Considering cardinal directions with cone-shaped subdivisions [45], in Figure 8(a), the relation between $A$ and $B$ is "east" with four divisions of space, while in Figure $8(\mathrm{~b})$, the relation between the same objects is "northeast" if we subdivide the space into eight sectors [51]. 


\section{Table 2}

The definitions of projective relations between points. The symbols $H P_{y z}^{+}$and $H P_{y z}^{-}$indicate the half-plane, respectively, to the right and left of the $y z$ oriented line. The notations $\left(-\infty_{y z}, y\right),[y, z]$ and $\left(z,+\infty_{y z}\right)$ represent the three intervals in which the $y z$ oriented line is subdivided by the two points $y$ and $z$ : these two points belong to the central interval.

\begin{tabular}{|c|c|c|c|}
\hline name & short name & drawing & definition \\
\hline collinear & $\operatorname{coll}(x, y, z)$ & 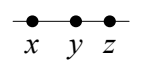 & $\exists$ line $l: x \in l, y \in l, z \in l$ \\
\hline aside & $\operatorname{aside}(x, y, z)$ & $\dot{x} \quad y_{\bullet} \bullet$ & $\neg \operatorname{coll}(x, y, z)$ \\
\hline rightside & $r s(x, y, z)$ & $\stackrel{\bullet}{x_{\bullet}} \stackrel{\bullet}{\longrightarrow}$ & $x \in H P_{y z}^{+}$ \\
\hline leftside & $l s(x, y, z)$ & $\stackrel{y}{x^{\bullet}} \stackrel{\bullet}{\longrightarrow}$ & $x \in H P_{y z}^{-}$ \\
\hline inside & $\operatorname{in}(x, y, z)$ & $x_{y} z$ & $x=y \wedge y=z$ \\
\hline outside & $o u(x, y, z)$ & $x \bullet \quad \dot{z}^{y}$ & $x \neq y \wedge y=z$ \\
\hline Jbetween & $b t(x, y, z)$ & 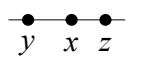 & $y \neq z \wedge x \in[y, z]$ \\
\hline nonbetween & $\operatorname{nonbt}(x, y, z)$ & 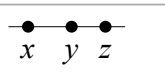 & $\operatorname{coll}(x, y, z) \wedge y \neq z \wedge x \notin[y, z]$ \\
\hline before & $b f(x, y, z)$ & $\stackrel{\bullet}{x} \quad \stackrel{\bullet}{*}:$ & $\operatorname{coll}(x, y, z) \wedge y \neq z \wedge x \in\left(-\infty_{y z}, y\right)$ \\
\hline after & $a f(x, y, z)$ & 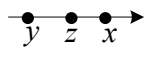 & $\operatorname{coll}(x, y, z) \wedge y \neq z \wedge x \in\left(z,+\infty_{y z}\right)$ \\
\hline
\end{tabular}

\section{Type and Dimension of Objects}

Spatial relations are also classified according to the type and dimension of the geometric objects to which they refer. The dimension 0,1, 2 corresponds to points, lines, and regions, respectively. Each of them can be of a different type: commonly, we distinguish between simple and complex objects (see Figure 9). The simple regions of the plane are regularly closed, with the interior and exterior connected; simple regions are therefore topologically equivalent to a disk. The simple lines are lines with only two limit points and no self-intersections (they are in a one-to-one match with an interval). Complex areas can have separate parts and holes. Complex lines can have more than two border points, separate parts, and intersections with themselves. The complex points are the union of several simple points.

Historically, most models of spatial relations have been initially developed for simple objects and then extended to complex objects. Depending on the type of geometry and the dimension, the models are able to distinguish a certain number of relations. For example, the 9IM is capable of distinguishing 33 topological relations between two simple lines. Table 3 is a summary of the number of topological relations for various models [23]. Relations between regions are indicated with $R / R$, between lines and regions with $\mathrm{L} / \mathrm{R}$, between points and regions with $\mathrm{P} / \mathrm{r}$, between lines with $\mathrm{L} / \mathrm{L}$, between points and lines with $\mathrm{P} / \mathrm{L}$ and between points with $\mathrm{P} / \mathrm{P}$.

The number of topological relations that models are able to distinguish for complex objects is of course larger. For example, for the 9IM, the number of different relations between two complex lines goes to 82 . In Table 4, there is a summary of the 9IM applied to complex objects [62]. As far as this axis is concerned, there is no work on spatial relations other than topological relations. 


\section{Figure 7}

Different granularities for qualitative distances: (a) two subdivisions of space; (b) three subdivisions of space.

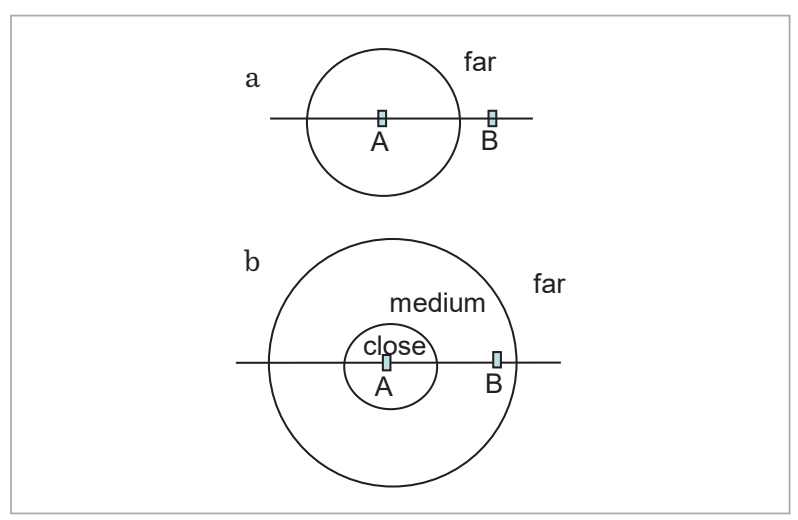

\section{Figure 8}

Different granularities for qualitative orientations: (a) four subdivisions of space; (b) eight subdivisions of space

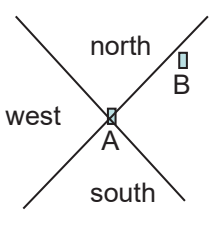

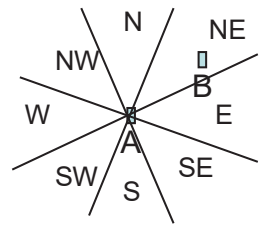

$\mathrm{b}$

\section{Dimension of the Embedding Space}

The dimension of the space where objects are defined is very important for the classification of spatial relations. The reason we are discussing this issue as last point is due to the fact that most of the work on spatial relations concerns the $2 \mathrm{D}$ plane. The use of other spaces is therefore a subject of research. Among the existing work on topology, we mention an extension of the 9IM to 3D space [68] and another approach that also considers the dimension of the parts of the objects [9]. The CBM has been extended to 3D space in [65]. With regard to projective relations, the 5-intersection model [19] was extended to 3D in [8].

In addition to $2 \mathrm{D}$ and 3D embedding spaces, there are other spaces that are important in some applications. For GIS applications, it would be interesting to study spatial relations on a spherical surface. There is not much work in this research direction, with the excep- tion of few contributions about topological relations [34] and projective relations [16]. In urban applications, it is important to examine 3D objects that are anchored to the Earth's surface with an added vertical direction: work on visibility relations between such objects has been preliminary carried out in $[41,43,64]$.

\section{Conclusion}

In this paper, we have described a conceptual framework for spatial relations modeling. Spatial relations find applications in many endeavors, from spatial databases to crowdsourced geoinformation [42, 56], from geographic information retrieval to indoor navigation [61]. The framework categorizes spatial relations in six independent criteria (orthogonal axes). The proposed categorization is important to place all the models in a common framework, to integrate different approaches, and to identify the areas in which there is still room to advance the research.

\section{Figure 9}

Examples of objects of different types and dimensions: (a) simple point; (b) simple line; (c) simple region, (d) complex point; (e) line with more than two boundary points and self-intersections; (f) region with separate parts and holes. For the sake of clarity, the point size is exaggerated

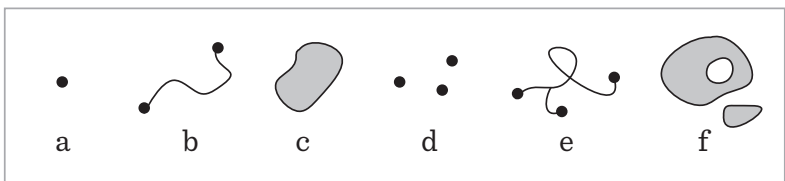

Table 3

Summary of topological relations for all models and for all dimensions between simple objects

\begin{tabular}{l|l|l|l|l|l|l|l}
\hline Model/group & $\mathbf{R} / \mathbf{R}$ & L/R & P/R & L/L & P/L & P/P & total \\
\hline 4IM & 8 & 11 & 3 & 16 & 3 & 2 & 43 \\
\hline 9IM & 8 & 19 & 3 & 33 & 3 & 2 & 68 \\
\hline $\mathrm{DEM}$ & 12 & 17 & 3 & 24 & 3 & 2 & 61 \\
\hline $\mathrm{DE}+9 \mathrm{IM}=\mathrm{CBM}$ & 12 & 31 & 3 & 36 & 3 & 2 & 87 \\
\hline
\end{tabular}

Table 4

Number of topological relations between two complex spatial objects for the 9IM

\begin{tabular}{|l|l|l|l|l|l|l|l}
\hline Model/group & R/R & L/R & P/R & L/L & P/L & P/P & total \\
\hline 9IM & 33 & 43 & 7 & 82 & 14 & 5 & 184 \\
\hline
\end{tabular}


With regard to the first axis, it is especially the user level that has been less investigated: a user ontology approach is suggested to bridge the gap with spatial relations at the geometric and computational levels. With regard to the second axis, it is the projective and metric relations that need more development, since topological relations have previously had a major role. Regarding cardinality, most of the past work has focused on binary relations, while higher cardinality deserves much attention. Regarding granularity,

\section{References}

1. Abella, A., Kender, J. R. From Images to Sentences via Spatial Relations. Proceedings of the Integration of Speech and Image Understanding: ICCV'99 Workshop, IEEE Computer Society, 1999, 117-146. https://doi. org/10.1109/ISIU.1999.824875

2. Bartie, P., Clementini, E., Reitsma, F. A Qualitative Model for Describing the Arrangement of Visible Cityscape Objects from an Egocentric Viewpoint. Computers, Environment and Urban Systems, 2013, 38, 21-34. https://doi.org/10.1016/j.compenvurbsys.2012.11.003

3. Berdier, C., Roussey, C. Urban Ontologies: The Towntology Prototype Towards Case Studies. Proceedings of Workshop of COST Action C21 Towntology - Ontologies for Urban Development: Interfacing Urban Information Systems, Université de Genève, 91-102.

4. Berretti, S., Del Bimbo, A., Vicario, E. Weighted Walkthroughs Between Extended Entities for Retrieval by Spatial Arrangement. IEEE Transactions on Multimedia, 2003, 5, 52-70. https://doi.org/10.1109/ TMM.2002.802833

5. Billen, R., Clementini, E. A Model for Ternary Projective Relations Between Regions. In Bertino, E., Christodoulakis, S., Plexousakis, D., Christophides, V., Koubarakis, M., Böhm, K., Ferrari, E. (Eds.), EDBT2004 - 9th International Conference on Extending DataBase Technology, Heraklion, Crete, Greece, March 14-18, 2004, Springer-Verlag: Berlin, 2004, 2992, 310-328. https:// doi.org/10.1007/978-3-540-24741-8_19

6. Billen, R., Clementini, E. Introducing a Reasoning System Based on Ternary Projective Relations. In Handling, Fisher, P. (Ed.), Developments in Spatial Data Handling, 11th International Symposium on Spatial Data Springer-Verlag: Berlin, 2005, 381-394. https:// doi.org/10.100r/3-540-26r72-7_29 more research is needed because often spatial relations models are defined at a coarse granularity level. Regarding the fifth axis (dimension and types of objects), it is necessary to extend models that were developed for $2 \mathrm{D}$ simple regions to other dimensions and complex regions and lines. Finally, most models considered the 2D plane as embedding space: other embedding spaces, such as the sphere and 3D space, have been little explored so far.

7. Billen, R., Clementini, E. Semantics of Collinearity Among Regions. In Meersman, R., Tari, Z., Herrero, P. (Eds.), On the Move to Meaningful Internet Systems 2005: OTM Workshops - 1st Int. Workshop on Semantic-Based Geographical Information Systems (SeBGIS’05), Agia Napa, Cyprus, October 31 - November 4, 2005, Springer-Verlag: Berlin, 2005, 3762, 1066-1076. https://doi.org/10.100r/115r5863_129

8. Billen, R., Clementini, E. Projective Relations in a 3D Environment. In Raubal, M., Miller, H., Frank, A., Goodchild, M. (Eds.), Geographic Information Science: 4th International Conference, GIScience 2006, Münster, Germany, September 20-23, 2006, Springer: Berlin, 2006, 4197, 18-32. https://doi.org/10.1007/11863939_2

9. Billen, R., Zlatanova, S., Mathonet, P., Boniver, F. The Dimensional Model: A Framework to Distinguish Spatial Relationships. In Richardson, D., van Oosterom, P. (Eds.), Advances in Spatial Data Handling - 10th International Symposium on Spatial Data Handling, Springer: Berlin, 2002, 285-298. https://doi.org/10.1007/9783-642-56094-1_21

10. Bloch, I. Fuzzy Relative Position Between Objects in Image Processing: A Morphological Approach. IEEE Transactions on Pattern Analysis and Machine Intelligence, 1999, 21, 657-664. https://doi.org/10.1109/34.7r77378

11. Bloch, I., Colliot, O., Cesar Jr., R. M. On the Ternary Spatial Relation "Between". IEEE Transactions on Systems, Man, and Cybernetics - Part B: Cybernetics, 2006, 36, 312-327. https://doi.org/10.1109/TSMCB.2005.857095

12. Cardenas, A. F., Ieong, I. T., Taira, R. K., Barker, R., Breant, C. M. The Knowledge-Based Object-Oriented PICQUERY+ Language. IEEE Transactions on Knowledge and Data Engineering, 1993, 5, 644-65\%. https://doi. org/10.1109/69.2347r76 
13. Casati, R., Varzi, A. Parts and Places: The Structures of Spatial Representations. MIT Press: Cambridge, MA, 248, 1999.

14. Cicerone, S., Clementini, E. Efficient Estimation of Qualitative Spatial Relations Based on the Weighted Walkthroughs Model. GeoInformatica, 2003, 7, 211227. https://doi.org/10.1023/A:1025148831131

15. Clementini, E. A Model for Uncertain Lines. Journal of Visual Languages \& Computing, 2005, 16, 271-288. https://doi.org/10.1016/j.jvlc.2004.11.003

16. Clementini, E. Projective Relations on the Sphere. In Song, I.-Y. (Ed.), Advances in Conceptual Modeling Challenges and Opportunities - ER 2008 Workshops - 2nd International Workshop on Semantic and Conceptual Issues in GIS (SeCoGIS 2008), Springer-Verlag: Berlin Heidelberg, 2008, 5232, 313-322. https://doi. org/10.1007/978-3-540-87991-6_38

17. Clementini, E. Ontological Impedance in 3D Semantic Data Modeling. In Kolbe, T. H., König, G., Nagel, C. (Eds.), International Archives of the Photogrammetry, Remote Sensing and Spatial Information Sciences XXXVIII-4, part W15 ISPRS: 5th 3D GeoInfo Conference, 3-4 November 2010, Berlin, Germany, 2010, 977-100.

18. Clementini, E. Directional Relations and Frames of Reference. GeoInformatica, 2013, 17, 235-255. https:// doi.org/10.1007/s10707-011-0147-2

19. Clementini, E., Billen, R. Modeling and Computing Ternary Projective Relations Between Regions. IEEE Transactions on Knowledge and Data Engineering, 2006, 18, 799-814. https://doi.org/10.1109/ TKDE.2006.102

20. Clementini, E., Billen, R. Projective Relations. In Shekhar, S., Xiong, H., Zhou, X. (Eds.), Encyclopedia of GIS, 2nd Edition, Springer International Publishing: New York, 2016. doi:10.1007/978-3-319-23519-6_1540-1pp. 1-10.

21. Clementini, E., Billen, R., Santic, M. Errata Corrige on "Modeling and Computing Ternary Projective Relations Between Regions". IEEE Transactions on Knowledge and Data Engineering, 2013, 25, 1196-1198. https:// doi.org/10.1109/TKDE.2011.239

22. Clementini, E., Cohn, A. G. RCC ${ }^{*}-9$ and CBM*. In Duckham, M., Pebesma, E., Stewart, K., Frank, A. U. (Eds.), Proceedings of Geographic Information Science, 8th International Conference, GIScience 2014, Vienna, Austria, September 24-26, 2014., Springer: Berlin, 2014, LNCS 8728, 349-365. https://doi.org/10.1007/978-3319-11593-1_23
23. Clementini, E., Di Felice, P. A Comparison of Methods for Representing Topological Relationships. Information Sciences, 1995, 3, 149-178.

24. Clementini, E., Di Felice, P. Topological Invariants for Lines. IEEE Transactions on Knowledge and Data Engineering, 1998, 10, 38-54. https://doi. org/10.1109/69.667085

25. Clementini, E., Di Felice, P. A Spatial Model for Complex Objects with a Broad Boundary Supporting Queries on Uncertain Data. Data \& Knowledge Engineering, 2001, 37, 285-305. https://doi.org/10.1016/ S0169-023X(01)00010-6

26. Clementini, E., Di Felice, P., Hernández, D. Qualitative Representation of Positional Information. Artificial Intelligence, 1997, 95, 317-356. https://doi.org/10.1016/ S0004-3702(97)00046-5

27. Clementini, E., Di Felice, P., van Oosterom, P. A Small Set of Formal Topological Relationships Suitable for End-User Interaction. In Abel, D., Ooi, B.C. (Eds.), Advances in Spatial Databases - Third International Symposium, SSD '93, Springer-Verlag: Berlin, 1993, 692, 277-295. https://doi.org/10.1007/3-540-56869-7_16

28. Clementini, E., Skiadopoulos, S., Billen, R., Tarquini, F. A Reasoning System of Ternary Projective Relations. IEEE Transactions on Knowledge and Data Engineering, 2010, 22, 161-178. https://doi.org/10.1109/ TKDE.2009.79

29. Cohn, A. G., Bennett, B., Gooday, J., Gotts, N. Qualitative Spatial Representation and Reasoning with the Region Connection Calculus. GeoInformatica, 1997, 1, 275-316. https://doi.org/10.1023/A:1009712514511

30. Cohn, A. G., Gotts, N. M. The 'Egg-Yolk' Representation of Regions with Indeterminate Boundaries. In Burrough, P. A., Frank, A. U. (Eds.), Geographic Objects with Indeterminate Boundaries, Taylor \& Francis: London, 1996, 2, 171-187.

31. Cohn, A. G., Hazarika, S. M. Qualitative Spatial Representation and Reasoning: An Overview. Fundamenta Informaticae, 2001, 46, 1-29.

32. Couclelis, H. People Manipulate Objects (but Cultivate Fields): Beyond the Raster-Vector Debate in GIS. Proceedings of Theories and Methods of Spatio-Temporal Reasoning in Geographic Space, Pisa, Italy, September 21-23, 1992, 65-777. https://doi.org/10.10077/3-54055966-3_3

33. Dehak, S. M. R., Bloch, I., Maître, H. Spatial Reasoning with Incomplete Information on Relative Position- 
ing. IEEE Transactions on Pattern Analysis and Machine Intelligence, 2005, 27, 1473-1484. https://doi. org/10.1109/TPAMI.2005.186

34. Egenhofer, M. J. Spherical Topological Relations. In Spaccapietra, S., Zimányi, E. (Eds.), Journal on Data Semantics III, Springer-Verlag: Berlin, 2005, 3534, 25-49. https://doi.org/10.1007/11496168_2

35. Egenhofer, M. J., Franzosa, R. D. Point-Set Topological Spatial Relations. International Journal of Geographical Information Systems, 1991, 5, 161-174. https://doi. org/10.1080/02693799108927841

36. Egenhofer, M. J., Franzosa, R. D. On the Equivalence of Topological Relations. International Journal of Geographical Information Systems, 1995, 9, 133-152. https://doi.org/10.1080/02693799508902030

37. Egenhofer, M. J., Herring, J. R. Categorizing Binary Topological Relationships Between Regions, Lines, and Points in Geographic Databases; Department of Surveying Engineering, University of Maine, Orono, ME, 1991.

38. Egenhofer, M. J., Mark, D. M. Naive Geography. In Frank, A. U., Kuhn, W. (Eds.), Spatial Information Theory: A Theoretical Basis for GIS - International Conference, COSIT'95, Springer-Verlag: Berlin, 1995, 988, 1-15. https://doi.org/10.1007/3-540-60392-1_1

39. Egenhofer, M. J., Sharma, J. Topological Relations Between Regions in R2 and Z2. In Abel, D., Ooi, B. C. (Eds.), Advances in Spatial Databases - Third International Symposium, SSD '93, Springer-Verlag: Berlin, 1993, 692, 316-336.

40. Favetta, F., Laurini, R. About Precision and Integrity in Visual Query Languages for Spatial Databases. Seventh International Conference on Database Systems for Advanced Applications (DASFAA 2001), 18-20 April 2001, Hong Kong, China, IEEE Computer Society: Los Alamitos, CA, 2001, 286-293. https://doi.org/10.1109/DASFAA.2001.916389

41. Fogliaroni, P., Clementini, E. Modeling Visibility in 3D Space: A Qualitative Frame of Reference. In Breunig, M., Al-Doori, M., Butwilowski, E., Kuper, P. V., Benner, J., Haefele, K. H. (Eds.), 3D Geoinformation Science: The Selected Papers of the 3D GeoInfo 2014, Springer: Berlin, 2015, LNG\&C, 243-258.

42. Fogliaroni, P., D’Antonio, F., Clementini, E. Data Trustworthiness and User Reputation as Indicators of VGI Quality. Geo-Spatial Information Science, 2018, 21, 213233. https://doi.org/10.1080/10095020.2018.1496556

43. Fogliaroni, P., Wallgrün, J. O., Clementini, E., Tarquini, F., Wolter, D. A Qualitative Approach to Localization and Navigation Based on Visibility Information. In Stewart Hornsby, K., Claramunt, C., Denis, M., Ligozat, G. (Eds.), Spatial Information Theory, 9th International Conference, COSIT 2009, Aber Wrac'h, France, September 2009, Springer: Berlin, 2009, 5756, 312-329. https://doi.org/10.1007/978-3-642-03832-7_19

44. Frank, A. U. MAPQUERY: Data Base Query Language for Retrieval of Geometric Data and their Graphical Representation. ACM Computer Graphics, 1982, 16, 199-207. https://doi.org/10.1145/965145.801281

45. Frank, A. U. Qualitative Reasoning About Distances and Directions in Geographic Space. Journal of Visual Languages and Computing, 1992, 3, 343-371. https://doi. org/10.1016/1045-926X(92)90007-9

46. Freksa, C. Temporal Reasoning Based on Semi-Intervals. Artificial Intelligence, 1992, 54, 199-22\%. https:// doi.org/10.1016/0004-3702(92)90090-K

47. Goyal, R., Egenhofer, M. J. The Direction-Relation Matrix: A Representation of Direction Relations for Extended Spatial Objects. Proceedings of UCGIS Annual Assembly and Summer Retreat, Bar Harbor, ME.

48. Güting, R. H. Geo-Relational Algebra: A Model and Query Language for Geometric Database Systems. In Schmidt, J. W., Ceri, S., Missikoff, M. (Eds.), Advances in Database Technology - EDBT'88, Proceedings of the International Conference on Extending Database Technology, March 14-18, 1988, Venice, Italy, Springer-Verlag: Berlin, 1988, 303, 506-527.

49. Güting, R. H. An Introduction to Spatial Database Systems. The VLDB Journal, 1994, 3, 357-400. https://doi. org/10.1007/BF01231602

50. Hernández, D. Maintaining Qualitative Spatial Knowledge. In Frank, A. U., Campari, I. (Eds.) Spatial Information Theory: A Theoretical Basis for GIS - European Conference, COSIT'93, Springer-Verlag: Berlin, 1993, 716, 36-53. https://doi.org/10.1007/3-540-57207-4_4

51. Hernández, D. Qualitative Representation of Spatial Knowledge. Springer-Verlag: Berlin, 804, 202, 1994. https://doi.org/10.1007/BFb0020328

52. Herring, J. TIGRIS: A Data Model for an Object-Oriented Geographic Information System. Computers and Geosciences, 1991, 18, 443-452. https://doi. org/10.1016/0098-3004(92)90074-2

53. Ishikawa, T., Montello, D. R. Spatial Knowledge Acquisition from Direct Experience in the Environment: Individual Differences in the Development of Metric Knowledge and the Integration of Separately Learned Places. Cognitive Psychology, 2006, 52, 93-129. https://doi.org/10.1016/j.cogpsych.2005.08.003

54. Klein, F. Vergleichende Betrachtungen Über Neuere Geometrische Forschungen. Bulletin of the New York 
Mathematical Society, 1893, 2, 215-249. https://doi. org/10.1090/S0002-9904-1893-00147-X

55. Lautenschütz, A.-K., Davies, C., Raubal, M., Schwering, A., Pederson, E. The Influence of Scale, Context and Spatial Preposition in Linguistic Topology. In Barkowsky, T., Knauff, M., Ligozat, G., Montello, D. R. (Eds.), Spatial Cognition V Reasoning, Action, Interaction International Conference Spatial Cognition 2006, September 24-28, 2006, Bremen, Germany, Springer: Berlin, 2007, 4387, 439-452.

56. Martella, R., Kray, C., Clementini, E. A Gamification Framework for Volunteered Geographic Information. In Bação, F., Santos, M. Y., Painho, M. (Eds.), AGILE 2015 - Geographic Information Science as an Enabler of Smarter Cities and Communities, Lecture Notes in Geoinformation and Cartography, Springer: Berlin, 7389, 2015. https://doi.org/10.1007/978-3-319-16787-9_5

57. OGC. Geometry Object Model. In OpenGIS Implementation Specification for Geographic Information - Simple Feature Access - Part 1: Common Architecture, 2011, 13-32.

58. Parent, C., Spaccapietra, S., Zimanyi, E. Conceptual Modeling for Traditional and Spatio-Temporal Applications The MADS Approach; Springer: Berlin, 466, 2006.

59. Portillo, M. C., Pomerantz, J. R., Zimmerman, S. Evaluating Grouping via Emergent Features: A Systematic Approach. Fifth Annual Meeting of the Vision Science Society, May 7, 2005, Sarasota, FL, 2005.

60. Retz-Schmidt, G. Various Views on Spatial Prepositions. AI Magazine, 1988, 9, 95-105.

61. Russo, D., Zlatanova, S., Clementini, E. Route Directions Generation Using Visible Landmarks. In Claramunt, C., Li, K.-J., Zlatanova, S. (Eds.), Sixth ACM SIGSpatial International Workshop on Indoor Spatial
Awareness (ISA 2014), ACM, 2014, 1-8. https://doi. org/10.1145/2676528.2676530

62. Schneider, M., Behr, T. Topological Relationships Between Complex Spatial Objects. ACM Transactions on Database Systems, 2006, 31, 39-81. https://doi. org/10.1145/1132863.1132865

63. Tarquini, F., Clementini, E. Spatial Relations Between Classes as Integrity Constraints. Transactions in GIS, 2008, 12, 45-57. https://doi.org/10.1111/j.14679671.2008.01134.x

64. Tarquini, F., De Felice, G., Fogliaroni, P., Clementini, E. A Qualitative Model for Visibility Relations. In Hertzberg, J., Beetz, M., Englert, R. (Eds.), 30th Annual German Conference on Artificial Intelligence (KI 2007), Springer, 2007, 4667, 510-513. https://doi. org/10.1007/978-3-540-74565-5_52

65. van Oosterom, P., Vertegaal, W., van Hekken, M., Vijlbrief, T. Integrated 3D Modelling Within a GIS. In Molenaar, M., Hoop, S. D. (Eds.), Avanced Geographic Data Modelling: Spatial Data Modelling and Query Languages for 2D and 3D Applications, Netherlands Geodetic Commission: Delft, The Netherlands, 40, 80-95, 1994.

66. Wallgrün, J. O., Frommberger, L., Wolter, D., Dylla, F., Freksa, C. A Toolbox for Qualitative Spatial Representation and Reasoning. Proceedings of Spatial Cognition $\mathrm{V}$ : Reasoning, Action, Interaction: International Conference Spatial Cognition, Bremen, Germany, 2006, 39-58.

67. Winter, S., Frank, A. U. Topology in Raster and Vector Representation. GeoInformatica, 2000, 4, 35-65. https://doi.org/10.1023/A:1009828425380

68. Zlatanova, S. On 3D Topological Relationships. In Ibrahim, M. T., Küng, J., Revell, N., (Eds.), Database and Expert Systems Applications, 11th International Conference, DEXA 2000, September 4-8, 2000, London, UK, Springer: Berlin, 1873, 913-919, 2000. 